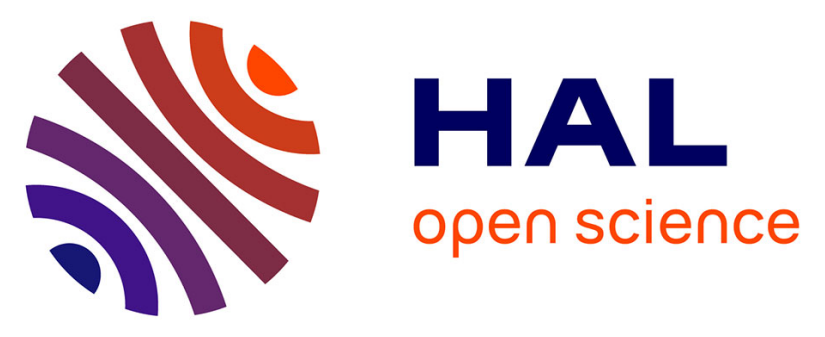

\title{
Hardware-Assisted Program Execution Integrity: HAPEI
}

Ronan Lashermes, Hélène Le Bouder, Gaël Thomas

\section{To cite this version:}

Ronan Lashermes, Hélène Le Bouder, Gaël Thomas. Hardware-Assisted Program Execution Integrity: HAPEI. NordSec 2018: 23rd Nordic Conference on Secure IT Systems, Nov 2018, Oslo, Norway. 10.1007/978-3-030-03638-6_25. hal-01978181

\section{HAL Id: hal-01978181 \\ https://hal.inria.fr/hal-01978181}

Submitted on 11 Jan 2019

HAL is a multi-disciplinary open access archive for the deposit and dissemination of scientific research documents, whether they are published or not. The documents may come from teaching and research institutions in France or abroad, or from public or private research centers.
L'archive ouverte pluridisciplinaire $\mathbf{H A L}$, est destinée au dépôt et à la diffusion de documents scientifiques de niveau recherche, publiés ou non, émanant des établissements d'enseignement et de recherche français ou étrangers, des laboratoires publics ou privés. 


\title{
Hardware-Assisted Program Execution Integrity: HAPEI
}

\author{
Ronan Lashermes ${ }^{1}$, Hélène Le Bouder ${ }^{2}$, and Gaël Thomas ${ }^{3}$ \\ 1 INRIA-RBA SED\&LHS, France \\ ronan.lashermes@inria.fr \\ 2 IMT-Atlantique, France \\ helene.le-bouder@imt-atlantique.fr \\ ${ }^{3}$ DGA-MI, France
}

\begin{abstract}
Even if a software is proven sound and secure, an attacker can still insert vulnerabilities with fault attacks. In this paper, we propose HAPEI, an Instruction Set Randomization scheme to guarantee Program Execution Integrity even in the presence of hardware fault injection. In particular, we propose a new solution to the multi-predecessors problem. This scheme is then implemented as a hardened CHIP-8 virtual machine, able to ensure program execution integrity, to prove the viability and to explore the limits of HAPEI.
\end{abstract}

Keywords: Program Execution Integrity, Control Flow Integrity, Hardware Fault Attacks, Instruction Set Randomization

\section{Introduction}

In order to ensure the security of an application, developers have to do every thing they can to reduce the number of bugs that could lead to vulnerabilities. But for the most critical applications, software must be proven correct. Yet one bug missed and an attacker can, in some cases, execute arbitrary code. Moreover, this bug can be absent in the binary but created at runtime with a hardware fault injection, breaking software proofs assumptions.

Motivation The problem is illustrated below with a simple example.

Listing 1.1. A simple loop in C.

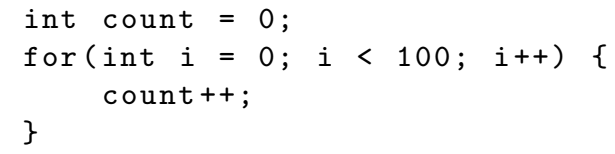

The assembly code corresponding with the loop in listing 1.1 can be seen in listing 1.2 
Listing 1.2. The same loop in x86 assembly.

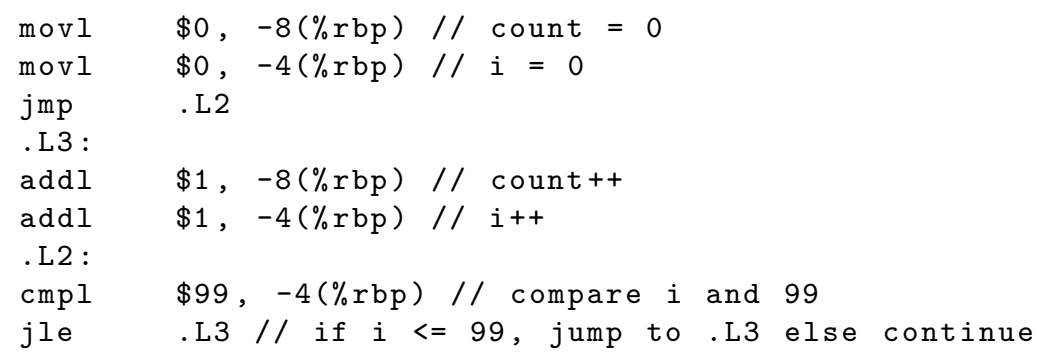

In this case the program execution must ensure:

- instructions are executed in order,

- it is not possible to jump to an arbitrary instruction of the loop. Only the "landing" instructions can be jumped to (first ones after L2 or L3).

- When executing a "landing" instruction, the previous state of the program must be correct. E.g. the program state is one of the two authorized ones (a proper definition of program state is given in section 4). This implies that the jumps are all legitimate.

- No instruction can be overwritten, no instruction can be skipped.

These guarantees must be valid even if the attacker is able to arbitrarily modify instructions at runtime. In this case, execution must stop to prevent further damages.

Contribution This work proposes HAPEI to ensure that the intended software is what is actually running on the chip. Inspired by SOFIA [13, the solution is a hardware Instruction Set Randomization (ISR) scheme that ensures Instructions Integrity (II) and Control Flow Integrity (CFI), even in the case of a Hardware Fault Attacks on Instructions (HFAoI). We demonstrate that we can harden a binary without any modification in the compilation chain with a CHIP-8 virtual machine implementation. It means that the Instruction Set Architecture (ISA) does not have to change in the compiler's view: HAPEI is transparent at the software level. During application installation (also called packing), the instructions are encrypted. The encryption scheme encodes the authorized program states and the transitions from one state to the next (effectively encoding the Control Flow Graph (CFG) . During execution, instructions are decrypted on-the-fly. The decryption is correct only if the program state is correct and the control flow graph is followed.

Organization In this paper, we start with the context and the necessary definitions in section 2. After a review of similar relevant works in section 3, the theory behind HAPEI is presented in section 4 A discussion on the security of the scheme follows in section 5. We propose an implementation detailed in section 6 and finally our conclusion is drawn in section 7 . 


\section{Context}

In order to specify and verify our proposal, we must precisely define the capacities of the attacker and the integrity guarantees we provide.

Attacker models Several models are considered:

- Code Injection Attack (CIA) an attacker tries to divert the control flow to execute its own malicious payload.

- Code-Reuse Attack (CRA) an attacker tries to execute a malicious payload composed by a sequence of legitimate pieces of programs (often called widgets in Return-Oriented Programming).

- Hardware Fault Attacks on Instructions (HFAoI) the attacker can edit the program, at runtime, by modifying instruction values.

- Hardware Fault Attacks on Data (HFAoD) the attacker can edit the program, at runtime, by modifying data values.

Integrities To protect against these attacks, the execution of the software must enforce guarantees:

- Control Flow Integrity (CFI) the control flow cannot be modified (no arbitrary jumps). The control flow follows the valid CFG

- Instructions Integrity (II) the instruction values must not be altered.

- Data Integrity (DI) the data handled by the program must not be altered.

- State Integrity (StI) the processor state (configuration, registers, program counter, ...) must not be altered.

Often, DI III and StI are considered together under the name of System Integrity (SI), Here we call Program Execution Integrity (PEI) the combination of CFI and II.

Our scheme, HAPEI, ensures PEI in order to protect against CIA, CRA and HFAol Yet to be complete, a solution should also ensure DI. In our opinion, one of the best solution would be to encrypt all data with one secret key per application. Since in the following we consider only one application executing, we suppose that data integrity is guaranteed at a higher level.

StI is probably the most difficult to guarantee in presence of an attacker with hardware fault injection means. It is also very implementation specific, we discuss in section 5.3 how an attack can be achieved on our implementation because StI is not guaranteed.

If numerous works discuss how to ensure integrities, most consider only CIA and CRA attacker models. Yet, hardware fault attacks are a reality and must be mitigated. Unfortunately, HFAol is a much more powerful attacker model and most previous schemes do not protect against it (cf section 3). 


\section{Previous works}

This work inherits from a long list of proposals to ensure CFI and SI in the literature. In this section, we present the most relevant works (that we know of) and show where they differ with HAPEI. In most cases, CFI and $[\mathrm{SI}$ are viewed as orthogonal and protected with different solutions.

CFI 3. consists in ensuring that the control flow cannot be tampered with. A large literature exists on the subject, a recent review article by Burrow et al. [4] compares many solutions. Paraphrasing Burrow et al. 4, ISAs usually have two forward control-flow transfers: jumps and routine calls. We consider separately direct jumps (where the destination address is static) and indirect jumps where the destination address can only be determined at runtime. Most CFI solutions try to verify that jumps can only reach legitimate addresses (forward edges). A special case is the RETURN instruction that jump to the value on top of the stack, to return from a routine call (backward edges). As a consequence, a part of the CFG can be determined statically, but another part cannot due to indirect jumps.

Abadi et al. 3 shows software CFI implementations: they propose code snippets to replace dangerous instructions (indirect jumps... ) in order to guarantee CFI.

Tice et al. [15] demonstrate a software solution that leverages the compiler to automatically insert the appropriate protections at jump sites (forward edges only). In particular, they tackle the problem caused by virtual method tables, necessary in some programming languages $($ e.g. $\mathrm{C}++)$ to enforce runtime polymorphism. In this situation, the method to call is decided at runtime and thus requires an indirect call.

Backward edges (e.g. RETURN instruction) are traditionally protected with a shadow stack [9]: the call stack is duplicated. On a RETURN instruction, the return address on both stacks are read then compared. If they differ, an alarm is triggered. Another possibility explored by Davi et al. [76] is to add instructions to the ISA for the sole purpose of validating function calls and returns. On any indirect function call, the processor switch to a particular state. The next instruction must be a special CFIBR label instruction in order to continue execution. The label is used to keep track of which functions are currently executing.

These methods are efficient but focus on preventing CRAs. Because they are more difficult to achieve, Hardware Fault Attacks are not considered. However, hardware fault attacks have been known for some time. Dehbaoui at al. [8] shows that electromagnetic pulses allow to recover a cryptographic secret key. Then Moro et al. 12 describes the faulting mechanism and how it translates in a software model. Hardware fault attacks can be generated by software. The recent CLKSCREW work by Tang et al. [14, where the authors modify a phone's energy and clock controller to inject faults, demonstrates such an attack. Another illustration is given by the RowHammer attack [16. These attacks are relevant and must be mitigated: HAPEI must ensure CFI even in the presence of HFAoI. 
CFI ensures that jumps, routine calls and returns are legitimate, but it does not prevent an attacker to alter any other instruction. New mitigation techniques should be used for that: II and DI must be ensured.

Most system integrity techniques rely on the encryption of memories, preferably with dedicated hardware. Danger at al. [5] introduce a new instruction to selectively randomize parts of a program. Closer to us, Hiscock et al. [10] propose a scheme that encrypt the whole application using a stream cipher. In order to deal with multi-predecessors (where one instruction may have several predecessors, thus breaking the stream pattern), the authors re-init the stream cipher.

When specifically applied to instructions, which must be therefore decrypted on-the-fly at execution, the technique is called Instruction Set Randomization (ISR [11. Without the secret key, the attacker is unable to alter an instruction and predict the result after decryption. One of the most complete solution, ensuring both CFI and II is SOFIA [13]. This work is the main inspiration for HAPEI.

In SOFIA, to ensure CFI we must encode the authorized state of the program. The solution, proposed in [13, is to mask the instructions with a key stream depending on the Program Counter $(P C)$ and the previous Program Counter $P C_{\text {prev }}$ (for the previous instruction). Let $i$ be an opcode (instruction value) and $i^{\prime}$ the corresponding encrypted opcode. Let $E_{k}$ be an symmetric encryption function with secret $k$.

$$
i^{\prime}=E_{k}\left(P C_{\text {prev }}\|P C\| \ldots\right) \oplus i
$$

This elegant solution ensure that an instruction can be decoded only if $P C$ and $P C_{\text {prev }}$ are correct. Effectively, it encodes all the possible successions of instructions and the correct instruction can be decoded only upon correct $P C$ and $P C_{\text {prev }}$ values. In order to ensure II, a Message Authentication Code (MAC) is computed and verified per batch of up to 6 instructions. The MAC value is stored as two words at the beginning of each block. If an instruction has two predecessors, a special case must be made: the multiplexor block. In this block, the two first words correspond to the encrypted MAC values for the two possible predecessors: $M_{1 e 1}^{\prime}=E_{k}\left(P C_{p r e v}^{1}\|P C\| \ldots\right) \oplus M 1$ and $M_{1 e 2}^{\prime}=$ $E_{k}\left(P C_{p r e v}^{2}\|P C\| \ldots\right) \oplus M 1$. The encrypted MAC value not used (corresponding to the wrong predecessor) must be skipped in a software transparent way.

We acknowledge the power of this solution, and build our own upon it. Our main issue with SOFIA is the separation between CFI and II. Since the CFI mechanism relies on the Program Counter and not on the instruction value, an additional mechanism is needed to ensure II. Finally the multiplexor block must deal with possible predecessors in a non trivial way. It modifies the control flow according to the actual predecessor where it should be predecessor agnostic (all legitimate predecessors must be dealt with in the same way).

Our paper proposes an new solution to these problems, by relaxing the efficiency requirements. 


\section{HAPEI}

\subsection{Phases}

In order to harden the application, a preparatory step is required to encrypt the instructions. Only then, the application can be executed.

Packing Packing is required to create the encrypted program, enriched with the necessary metadata, following the scheme detailed below. It must be done on the final device, since it requires a device-specific secret key $k$.

Execution During execution, the encrypted instructions are deciphered on-thefly and executed. The decryption can only occur if the program state is correct.

\subsection{Program Execution Integrity}

SOFIA encodes the state of the program as the succession of $P C_{\text {prev }}$ and $P C$. We propose instead to encode the state of the program as the history of all previous executed instructions. Our proposal does not depend on the $P C$ value (apart when encoded in an instruction value). As such, the machine code is ensured to be executed correctly: instructions integrity is ensured together with control flow integrity.

Secondly, it becomes easy to check at anytime during execution that the current state of the program is valid.

We suppose that the Control Flow Graph (CFG) of our program is perfectly defined at compile time. There is no ambiguity on the destination address of jumps and calls. This condition is trivially satisfied if there are no indirect jumps or calls in our program. In the other case, it can be more tricky.

Let $a c c_{n}$ (standing for accumulator at instruction $n$ ) be a value representing the state of the program when instruction $i_{n}$ is about to be executed ( $n$ uniquely identify one instruction). $i_{n}$ and $a c c_{n}$ can be seen as values in $\mathbb{F}_{2^{w}}$ and $\mathbb{F}_{2^{b}}$ respectively for some $w$ and $b . b$ is the instruction size (considered fixed) and $w$ is the security parameter.

Bootstrap To bootstrap the encoding, one has to use an initialization vector $I V$ as input for the first executed instruction.

$$
a c c_{\text {init }}=H M A C_{k}(I V)
$$

$a c c_{\text {init }}$ is considered as a predecessor program state to the entry instruction. It may be used in a multi-predecessor scheme or in the 1-predecessor one.

1-predecessor The easy case is the 1-predecessor case. Our program snippet is a succession of instructions $\left[i_{1}, i_{2}, \cdots, i_{n}, \cdots\right]$ where all instructions are executed in order.

The instructions are encoded as

$$
i_{n}^{\prime}=C\left(a c c_{n}\right) \oplus i_{n},
$$


where $C$ is a compression function: a projection from $\mathbb{F}_{2^{b}}$ to $\mathbb{F}_{2^{w}}$. $C$ must ensure that $x$ cannot be deduced from $C(x)$.

Obviously the state of the program must be updated, using secret key $k$ :

$$
a c c_{n+1}=H M A C_{k}\left(a c c_{n} \| i_{n}\right) .
$$

You can see that the state of the program is encoded with a hash chain depending on all previous instructions. The encoded instruction $i_{n}^{\prime}$ can only be decoded when the previous state of the program $a c c_{n}$ is correct. This is possible only when instruction $i_{n}$ is due. Decoding necessitates the same operations:

$$
\begin{aligned}
a c c_{n} & =H M A C_{k}\left(a c c_{n-1} \| i_{n-1}\right), \\
i_{n} & =C\left(a c c_{n}\right) \oplus i_{n}^{\prime} .
\end{aligned}
$$

2-predecessors, a naive and limited solution Most programs necessitate branching. As a consequence, some instructions have 2 predecessors ( 2 possible previous instructions at two different locations in the program).

As a consequence the previous state of the program may have 2 different values: $a c c_{n}^{1}$ or $a c c_{n}^{2}$. 1 out of the 2 possible values is required to decode $i_{n}$.

Let $\Sigma=a c c_{n}^{1} \oplus a c c_{n}^{2}$, we can encode our instruction as:

$$
\left\{\Sigma, i_{n}^{\prime}=C\left(a c c_{n}^{1} \cdot a c c_{n}^{2}\right) \oplus i_{n}\right\},
$$

i.e. the previous state is encoded as $a c c_{n}^{1} \cdot a c c_{n}^{2}$.

Two cases are possible: the previous state is $a c c_{n}=a c c_{n}^{1}$ or $a c c_{n}=a c c_{n}^{2}$. Either case, the decoding is:

$$
i_{n}=C\left(a c c_{n} \cdot\left(a c c_{n} \oplus \Sigma\right)\right) \oplus i_{n}^{\prime}
$$

Yet this scheme has a huge weakness: it is impossible to encrypt the program if cycles are present in the control flow. E.g. a loop's first instruction has two predecessors $a c c_{n}^{1}$ and $a c c_{n}^{2}$ where $a c c_{n}^{2}$ is the state of the program at the end of the loop. Then it becomes infeasible to compute $a c c_{n}^{2}$ : it depends on $a c c_{n}^{1} \cdot a c c_{n}^{2}$. The self-reference cannot be solved, since in this case it would violate Hashbased Message Authentication Code (HMAC) security requirements: one should not be able to find a preimage given an output.

So this scheme works only if the control flow graph is a Direct Acyclic Graph (DAG) which is very limiting in real life scenarios. Instead two solutions (A and B) are proposed below with different security implications developed in section 5 .

p-predecessors, solution $A$ It is possible to generalize in order to allow up to $p$ predecessors for an instruction and for a control flow with cycles.

In order to allow cycles, we must "rebase" our program state for all instructions having several predecessors. In this case, the program state is a new uniformly random value (noted $r$ below). The problem is now to map valid predecessor states to this same new state.

Let $r$ be a random value in $\mathbb{F}_{2^{b}}$. Let $a c c_{n}^{i}, i \in \llbracket 1, p \rrbracket$ be the allowed previous accumulator values for current instruction $i_{n}$. A polynomial $P$ can be defined 
such that $\forall i \in \llbracket 1, p \rrbracket, P\left(a c c_{n}^{i}\right)=r$ using Lagrange interpolation. Since the generated polynomial is minimal, it is constant if we do not define an additional point. $P(0)=d$ for $d$ a random value (different than $r$ ) in $\mathbb{F}_{2^{b}}$.

The $p$ coefficients of $P$ are stored as program metadata with the corresponding instruction $i_{n}$. At packing, HAPEI encrypts with $i_{n}^{\prime}=C(r) \oplus i_{n}$. To decrypt instruction $i_{n}$, we use $a c c_{n}=P\left(a c c_{n-1}\right)$. Note that the polynomial evaluation replaces the HMAC call.

p-predecessors, solution $B \mathbb{F}_{2^{b}}$ can be decomposed in different subgroups $\mu_{p}$ where

$$
\forall x \in \mu_{p}, x^{p}=1
$$

(subgroup of $p$ th-root of unity). Such subgroups exist for all $p$ such that $p \mid 2^{b}-1$.

For all valid $p$ (depending on $b$ ), we can define a scheme that allows $p$ predecessors. For example, $b=16$ allows a scheme with 5 predecessors ( $p=5$ divides $\left.65535=2^{16}-1\right)$.

Let $a c c_{n}^{i}, i \in \llbracket 1, p \rrbracket$ be the allowed previous accumulator values for current instruction $i_{n}$. Let $r$ be a random value in $\mathbb{F}_{2^{b}}$. Let $m \in \mu_{p}$ be a generator value for the subgroup. We construct a polynomial $P$ (in $\mathbb{F}_{2^{b}}$ ) using Lagrange interpolation such that $\forall i \in \llbracket 1, p \rrbracket, P\left(a c c_{n}^{i}\right)=r \cdot m^{i}$. The $p-1$ coefficients of $P$ are stored as program metadata with the corresponding instruction $i_{n}$. At packing, HAPEI encrypts with $i_{n}^{\prime}=C\left(r^{p}\right) \oplus i_{n}$. To decrypt instruction $i_{n}$, we use $a c c_{n}=P\left(a c c_{n-1}\right)^{p}$. Indeed, by construction

$$
\forall i \in \llbracket 1, p \rrbracket, P\left(a c c_{n}^{i}\right)^{p}=\left(r \cdot m^{i}\right)^{p}=r^{p} \cdot\left(m^{p}\right)^{i}=r^{p} .
$$

In this scheme, polynomials have degree $p-1$ instead of $p$ in solution A: the memory overhead is lower.

Ensuring Instruction Integrity To check the III it is enough to check an $a c c_{n}$ against a truth value pre-generated at packing time. The more frequent the check, the sooner a tempering is detected but the bigger is the required metadata.

A second strategy is to have valid instructions forming a set $I_{v} \subset \mathbb{F}_{2^{w}}$. If $\operatorname{card}\left(I_{v}\right)<<\operatorname{card}\left(\mathbb{F}_{2^{w}}\right)$ a wrongly decoded instruction will have a very low probability of belonging to $I_{v}$, of being valid.

\subsection{Key management}

In this scheme, the component responsible for managing the secret key $k$ is critical. In most cases, the binary encryption cannot be performed at compilation on the binary provider machine since it would requires to ship the (then shared) secret along with the binary. As a consequence, any Instruction Set Randomization (ISR) scheme using a secret key must have a packing phase that transform an unmodified binary (or extended with the CFG information) into a hardened one. 
The only other possibility is for the binary provider to encrypt the application for each intended recipient, then to use public-key cryptography to share the corresponding secret key with the targeted hardware.

\subsection{Limitations}

Apart from the performances overhead, our solution has severe limitations. Since the CFG must be perfectly known at packing time, indirect jumps and calls should be avoided. In particular, the scheme is not compatible with virtual method tables required for runtime polymorphism in several languages $(\mathrm{C}++$, java, ... ). Additionally, the scheme is tailored for self-contained applications. If the program must call external code (shared library, OS system call), things do not play well. How to lift these limits should be investigated by the community.

\section{Security Assessment}

In this section, the security of the solution is analysed. As with most equivalent schemes, the details are critical. In section 5.1, we discuss about the security of the proposed schemes. In section 5.2, we analyse the security problems due to the use of a stream cipher and how to overcome it. Finally, in section 5.3 , the limits of the Program Execution Integrity (PEI) guarantees are shown.

\subsection{Scheme security}

The scheme security relies on the secrecy of the key stream, the accumulator values must remain secret. Can the attacker gain information on one accumulator value, given she knows the encrypted instructions, the clear instructions (in the most advantageous case for her) and the polynomials ? If she learn a given $a c c_{n-1}$, then no information is gained on $a c c_{n}$ without the knowledge of the secret key $k$ per the cryptographic properties of the HMAC

First, she can deduce $C\left(a c c_{n}\right)=i_{n}^{\prime} \oplus i_{n}$. If $C$ is a cryptographically secure hash function, then no information is gained on $a c c_{n}$. Lower constraints on $C$ are possible, since we only care about the correct preimage security: the attacker must find the correct preimage, not just a satisfying one.

p-predecessors, solution $A$ Let $i_{n}$ be a $p$-predecessors instruction:

$$
\exists P \in \mathbb{F}_{2^{b}}[X] \mid \forall i \in \llbracket 1, p \rrbracket, P\left(a c c_{n}^{i}\right)=r
$$

with $r$ a random value. $P$ is a public non-constant polynomial but all $a c c_{n}^{i}$ and $r$ are secret.

Knowing $P$, the attacker cannot find any $a c c_{n}^{i}$ nor $r: r$ can be any value in $\mathbb{F}_{2^{b}}$ and for most $r$ she can find corresponding valid $a c c_{n}^{i}$. Yet if she learn $r$, then finding the roots of $P(X)-r$ is trivial. If she learn a given $a c c_{n}^{i}$, then she can compute $r=P\left(a c c_{n}^{i}\right)$, then find the other accumulator values. As a consequence, 
a polynomial links all corresponding secrets together. If one value is discovered, all the others are too.

A same accumulator value can be used as a legitimate input to several polynomials. Yet the resulting systems of equation are always underdetermined. There is one unknown per polynomial corresponding to the random $r$ value, plus at least 1 secret $a c c_{n}^{i}$ value. But there again, in this case all secrets are linked: discovering one may mean discovering the others.

The problem with solution $\mathrm{A}$ is that $P$ is constructed in a very specific way: Lagrange interpolation ensures that $P(X)-r$ has $p$ distinct roots $(P$ has degree $p$ ), the maximum possible. The attacker can use the peculiarity to gain information on $r$ and $a c c_{n}^{i}, \forall i$. Given a random polynomial $Q$ of degree $p$, the probability that $Q$ has $p$ distinct roots corresponds to the number of combinations to distribute $p$ roots over $2^{b}$ values divided by the total number of polynomials of degree $p$.

$$
\frac{\left(\begin{array}{c}
2^{b} \\
p
\end{array}\right)}{\left(2^{b}\right)^{p+1}} \text {. }
$$

Since in our case, $p<<2^{b}$, equation $(12)$ becomes

$$
\frac{1}{p !\left(2^{b}\right)^{p+1}} \text {. }
$$

As a conclusion, a proportion of $\frac{1}{p !}$ random polynomials have $p$ roots. The greater the $p$, the better for the attacker that becomes able to discriminate $r$. In most cases, $p$ is low and $2^{b}$ is high $(b \geq 128)$ so the security should not be compromised since the attacker cannot possibly enumerate all possible $r$.

p-predecessors, solution $B$ This possibility for the attacker to discriminate $r$ given $P$ is the main motivation for the alternative solution B. In this proposition, $r$ is not a special value with respect to $P: P(X)-r \cdot m^{i}$ may have any number of roots $(\geq 1)$. But then, it means that additional roots may be considered valid program states. Some random illegitimate accumulator values could be mapped to the legitimate one. Since the attacker should not be able to control the accumulator value, the security is not compromised.

Finally, the choice between solutions A and B depends on the attacker model: if she can control $a c c_{n}$, then solution A must be chosen. If not but she has a huge computation power, solution B should be preferred.

\subsection{Differential Attack}

If the attacker is able to see the plain/decrypted instructions (or deduce from observed behaviour), she can execute one arbitrary instruction $i_{a}$.

$$
i_{n}^{\prime} \oplus e=C\left(a c c_{n}\right) \oplus i_{n} \oplus e
$$



is

To execute $i_{a}$, simply choose $e=i_{n} \oplus i_{a}$. But the next state of the program

$$
a c c_{n+1}=H M A C_{k}\left(a c c_{n} \| i_{a}\right)
$$

which is unpredictable for the attacker by the required properties of $H M A C_{k}$. This attack is present in all schemes using a key stream (xoring a secret data with the text).

The mitigation is to wait for $i_{n+1}$ valid decryption before executing $i_{n}$. In this case, if the attacker tries to force execution of $i_{a}$ instead of $i_{n}$, II detects the bad behaviour (cf section 4.2).

\subsection{Multi-successors attack}

In the proposed scheme, several instructions can have the same associated program state. An example is given in listing 1.3

Listing 1.3. A pseudo assembly program

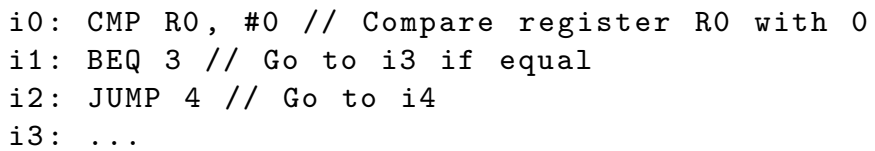

In this example, the possible transitions from instruction $i_{1}$ are $i_{1} \Rightarrow i_{2}$ or $i_{1} \Rightarrow i_{3} . i_{1}$ has two successors but both $i_{2}$ and $i_{3}$ have one predecessor. As a consequence, $a c c_{2}=a c c_{3}$ and encrypted instructions differential is conserved: $i_{2}^{\prime} \oplus i_{3}^{\prime}=i_{2} \oplus i_{3}$.

The attacker can switch these instructions at will and they will be correctly decoded. A mitigation would require to includes a unique identifier in the accumulator update formula:

$$
a c c_{n+1}=H M A C_{k}\left(a c c_{n}\left\|i_{n}\right\| n\right) .
$$

But such an attack does not break Program Execution Integrity the execution where the attacker switches the instructions is indistinguishable from a legitimate execution apart from instruction addresses. The program is semantically correct. And if the next instructions do not correspond to a legitimate program execution, they cannot be correctly decrypted. In conclusion, this attack illustrates the limits of the $\mathrm{PET}$ guarantees. The Program Counter $P C$ is part of the processor state: $\mathrm{StI}$ is the guarantee that should prevent this attack.

\section{Implementation}

In order to test HAPEI, we implement it by modifying a CHIP-8 virtual machine to run hardened programs. The sources for the reference and the hardened implementations can be found at https://gitlab.inria.fr/rlasherm/HAPEI. Licenses are MIT for software and CC-BY-4.0 for non-software. 


\subsection{CHIP-8}

CHIP-8 is an Instruction Set Architecture ISA initially intended to be run in a virtual machine on 8-bits microcomputers (from the 1970s). Its purpose is to run the same video games on different hardware. It is a good candidate for a hardened implementation because of its simplicity: 35 instructions with only 1 indirect branch instruction. Binaries (called roms in the video game emulation tradition) can be freely found on the internet. Additionally, its age means that it can easily be run on any modern computer, even with additional cryptographic computations, in real-time.

Our goal is therefore to run these roms in a hardened virtual machine. A simulated fault injection process, a key press modifies the next opcode by a randomly valid one, must be detected. In order to validate the hardened implementation, we compare it to a reference implementation (without the hardening) and compare the behaviours.

The implementation is modularized: chip8lib contains all common structure definitions and the machinery to parse opcodes into instructions (sum type values). chip8ref is the reference implementation, able to run, display and interact with emulated video games. chip8hard is the hardened implementation, it packs the current rom at startup then executes its encrypted version according to the scheme presented in this paper (solution A).

\subsection{Reference implementation}

The two implementations have been done in the rust language. Rust has great performances and allows a simple representation of the virtual machine by using sum types. The implementation has been inspired by the previous work at [2], but modularized to factor code between the reference and hardened implementations. The virtual machine is a 8-bit machine (word size) with 16-bit addresses.

\subsection{Hardened implementation}

Packing The hardened implementation must pack the application before execution. This step requires a precise control flow graph extraction. This extraction is done in a classical way. First we define a method $c f g$ next that given an instruction, its address and the call stack (stack to keep track of routine calls and returns) return all addresses that can possibly be executed next (and update the call stack). Then starting from the first address, we recursively call $c f g \_n e x t$ on the next instruction for all possible call stacks. Meaning that if the next instruction has already been included in the CFG previously but the call stack is different than the one during the previous CFG inclusion, we continue the analysis with the new call stack.

The difficulty lies in indirect branches that make CFG extraction difficult. In the CHIP-8, there is only one such instruction JP V0, addr that jumps to address addr plus the content of register Vo (8-bit register). In our CFG extraction, we consider that the possible successors for this instruction are all addresses between addr and addr +255 . Fortunately, all roms do not use this instruction. 
Once the CFG has been extracted, we compute all accumulator values (program states), polynomials and finally encrypt our instructions in the following order:

1. acc $_{\text {init }}$ from IV.

2. For all multi-predecessors instructions, draw a new random accumulator value. $a c c_{\text {init }}$ is a predecessor for the entry instruction.

3. Compute recursively all remaining accumulator values.

4. Compute and store polynomials for all multi-predecessors instructions.

5. For all instructions, encrypt it using corresponding accumulator value.

6. Delete all accumulator values, we have to compute them on-the-fly at execution.

Execution At execution, the binary is already encrypted. At each tick of the virtual machine, the following actions are performed in order to execute instruction $i_{n}$ at address $P C$ :

1. Is there a polynomial $P$ associated with address $P C$ ?

2. If yes, then its a multi-predecessors case: update the accumulator state $a c c=$ $P(a c c)$.

3. If there is no polynomial, then simply update the accumulator with the HMAC function: $a c c=H M A C_{k}\left(\right.$ acc $\left.\| i_{\text {previous }}\right)$

4. Then decrypt the instruction to be executed: $i_{n}=i_{n}^{\prime} \oplus C(a c c)$.

5 . If $i_{n}$ is valid we can execute it, in the other case we are under attack.

Only one accumulator value must be remembered throughout the computation, lowering the cost of our solution. This cost is both a big performance hit due to the on-the-fly decryption and accumulator update, and a memory overhead required to store the polynomials. Since our implementation is a virtual machine, the performance overhead cannot be meaningfully measured. But the memory overhead can be precisely measured as shown on table 1 .

In this table, the hardening is performed for a set of binaries found in 1 . We can see that the memory requirements at the 128-bit security level (size of one field element) is important: more memory is required to store the polynomials than the initial binary size.

Additionally, the roms are run in the reference virtual machine and in the hardened virtual machine to confirm functional equivalence. Then a simulated hardware fault injection mechanism is inserted. When a specific key is pressed, the next opcode is replaced in memory with a random valid opcode. On the reference implementation, the results of this fault injection is unpredictable: strange patterns are displayed on screen, nothing happen, another game screen is unlocked or we get a crash. In the hardened machine, the fault injection means that all subsequent instructions will be wrongly decoded: a crash always follows because of an invalid instruction value. 
Table 1. Hardening memory usage for a set of CHIP-8 roms found in 1] (solution A).

\begin{tabular}{|c|c|c|c|c|c|}
\hline ROM name & $\begin{array}{c}\text { ROM } \\
\text { byte size }\end{array}$ & $\begin{array}{c}\text { Instructions } \\
\text { count }\end{array}$ & $\begin{array}{c}\text { Polynomials } \\
\text { count }\end{array}$ & $\begin{array}{c}\text { Field } \\
\text { elements } \\
\text { count }\end{array}$ & $\begin{array}{c}\text { Polynomials } \\
\text { byte size } \\
\text { (128-bit) }\end{array}$ \\
\hline INVADERS & 1283 & 202 & 28 & 99 & 1584 \\
GUESS & 148 & 49 & 8 & 25 & 400 \\
KALEID & 120 & 59 & 10 & 32 & 512 \\
CONNECT4 & 194 & 67 & 5 & 19 & 304 \\
WIPEOFF & 206 & 101 & 15 & 47 & 752 \\
PONG2 & 264 & 126 & 19 & 60 & 960 \\
15PUZZLE & 384 & 116 & 17 & 54 & 864 \\
TETRIS & 494 & 189 & 32 & 106 & 1696 \\
BLINKY & 2356 & 856 & 84 & 310 & 4960 \\
VBRIX & 507 & 218 & 27 & 93 & 1488 \\
SYZYGY & 946 & 414 & 44 & 149 & 2384 \\
BRIX & 280 & 134 & 17 & 57 & 912 \\
TICTAC & 486 & 194 & 23 & 89 & 1424 \\
MAZE & 34 & 13 & 3 & 10 & 160 \\
PUZZLE & 184 & 87 & 10 & 34 & 544 \\
BLITZ & 391 & 121 & 15 & 47 & 752 \\
VERS & 230 & 103 & 24 & 73 & 1168 \\
PONG & 246 & 117 & 18 & 57 & 912 \\
UFO & 224 & 106 & 15 & 48 & 768 \\
TANK & 560 & 236 & 42 & 139 & 2224 \\
MISSILE & 180 & 75 & 12 & 37 & 592 \\
HIDDEN & 850 & 258 & 24 & 81 & 1296 \\
MERLIN & 345 & 124 & 13 & 45 & 720 \\
\hline & & & & & \\
\hline
\end{tabular}

\section{Conclusion}

In this paper, a solution to ensure Program Execution Integrity is presented. More precisely, Control Flow Integrity and Instructions Integrity are guaranteed against Code Injection Attack Code-Reuse Attack and Hardware Fault Attacks on Instructions. This solution uses the program state, a hash chain of all previously executed instructions, in order to encrypt the program. Correct decryption can only be achieved if the program state is correct.

The difficulty lies in the multi-predecessors case: how to handle the stream cipher when an instruction has several predecessors? Here, the program state is reinitialized with a random value and a polynomial is computed that maps all previous program states to this new value.

An implementation has been proposed as a CHIP- 8 virtual machine. It shows that the memory overhead is important and validates that the proposed scheme is functional.

Further work can be done to optimize the performances: can we find better mapping function than polynomials ? Is there a more compact representation of the program state, offering the same security level ? 
This work shows that instruction set randomization has a lot to offer in order to provide guarantees at the hardware level.

\section{References}

1. CHIP-8 games pack, https://www.zophar.net/pdroms/chip8.html

2. Mike zaby's CHIP-8 implementation, https://github.com/mikezaby/chip-8.rs

3. Abadi, Martín and Budiu, Mihai and Erlingsson, Ulfar and Ligatti, Jay: Controlflow integrity principles, implementations, and applications 13(1), 1-40, http:// portal. acm.org/citation. cfm?doid=1609956.1609960

4. Burow, N., Carr, S.A., Nash, J., Larsen, P., Franz, M., Brunthaler, S., Payer, M.: Control-flow integrity: Precision, security, and performance 50(1), 1-33, http: //dl.acm.org/citation. cfm?doid=3058791.3054924

5. Danger, J.L., Guilley, S., Praden, F.: Hardware-enforced protection against software reverse-engineering based on an instruction set encoding. pp. 1-11. ACM Press, http://dl .acm.org/citation.cfm?doid=2556464.2556469

6. Davi, L., Hanreich, M., Paul, D., Sadeghi, A.R., Koeberl, P., Sullivan, D., Arias, O., Jin, Y.: HAFIX: hardware-assisted flow integrity extension. pp. 1-6. ACM Press, http://dl.acm.org/citation.cfm?doid=2744769.2744847

7. Davi, L., Koeberl, P., Sadeghi, A.R.: Hardware-assisted fine-grained control-flow integrity: Towards efficient protection of embedded systems against software exploitation. pp. 1-6. IEEE, http://ieeexplore.ieee.org/document/6881460/

8. Dehbaoui, A., Dutertre, J.M., Robisson, B., Tria, A.: Electromagnetic transient faults injection on a hardware and a software implementations of AES. pp. 7-15. IEEE, http://ieeexplore.ieee.org/document/6305224/

9. Frantzen, M., Shuey, M.: StackGhost: Hardware facilitated stack protection. USENIX

10. Hiscock, T., Savry, O., Goubin, L.: Lightweight software encryption for embedded processors. pp. 213-220. IEEE, http://ieeexplore.ieee.org/document/ 8049788/

11. Kc, G.S., Keromytis, A.D., Prevelakis, V.: Countering code-injection attacks with instruction-set randomization p. 10

12. Moro, N., Dehbaoui, A., Heydemann, K., Robisson, B., Encrenaz, E.: Electromagnetic fault injection: Towards a fault model on a 32-bit microcontroller. pp. 77-88. IEEE, http://ieeexplore.ieee.org/document/6623558/

13. Ruan de Clercq, Ronald De Keulenaer, Bart Coppens, Bohan Yang, Pieter Maene, Koen de Bosschere, Bart Preneel, Bjorn de Sutter, Ingrid Verbauwhede: SOFIA: Software and control flow integrity architecture. IEEE

14. Tang, A., Sethumadhavan, S., Stolfo, S.: CLKSCREW: Exposing the perils of security-oblivious energy management. In: Proceedings of the Second Workshop on Real, Large Distributed Systems. USENIX, OCLC: 255334142

15. Tice, Caroline and Roeder, Tom and Collingbourne, Peter and Checkoway, Stephen and Erlingsson, Úlfar and Lozano, Luis and Pike, Geoff: Enforcing forward-edge control-flow integrity in GCC \& LLVM p. 15

16. van der Veen, V., Fratantonio, Y., Lindorfer, M., Gruss, D., Maurice, C., Vigna, G., Bos, H., Razavi, K., Giuffrida, C.: Drammer: Deterministic rowhammer attacks on mobile platforms. pp. 1675-1689. ACM Press, http://dl.acm.org/citation. cfm?doid=2976749.2978406 